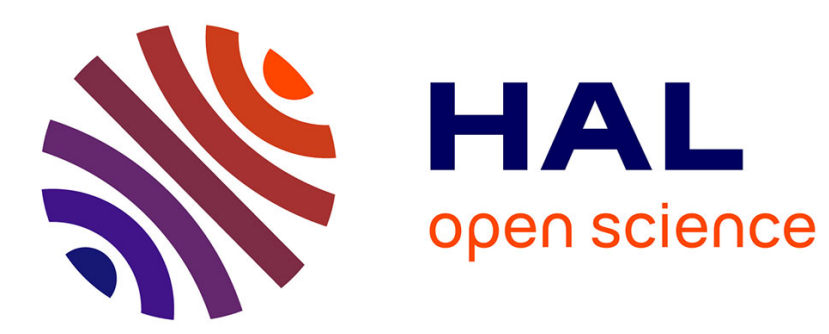

\title{
Humanoid Flexibility Deformation Can Be Efficiently Estimated Using Only Inertial Measurement Units and Contact Information
}

\author{
Mehdi Benallegue, Florent Lamiraux
}

\section{- To cite this version:}

Mehdi Benallegue, Florent Lamiraux. Humanoid Flexibility Deformation Can Be Efficiently Estimated Using Only Inertial Measurement Units and Contact Information. 2014 IEEE-RAS International Conference on Humanoid Robots, Nov 2014, Madrid, Spain. hal-01079797

\section{HAL Id: hal-01079797 https://hal.science/hal-01079797}

Submitted on 3 Nov 2014

HAL is a multi-disciplinary open access archive for the deposit and dissemination of scientific research documents, whether they are published or not. The documents may come from teaching and research institutions in France or abroad, or from public or private research centers.
L'archive ouverte pluridisciplinaire HAL, est destinée au dépôt et à la diffusion de documents scientifiques de niveau recherche, publiés ou non, émanant des établissements d'enseignement et de recherche français ou étrangers, des laboratoires publics ou privés. 


\title{
Humanoid Flexibility Deformation Can Be Efficiently Estimated Using Only Inertial Measurement Units and Contact Information
}

\author{
Mehdi Benallegue ${ }^{1,2}$ and Florent Lamiraux ${ }^{1,2}$
}

\begin{abstract}
Most robots are today controlled as being entirely rigid. But often, as for HRP-2 robot, there are flexible parts, intended for example to absorb impacts. The deformation of this flexibility changes the configuration of the robot, particularly in orientation. Nevertheless, robots have usually inertial sensors (IMUs) to reconstruct their orientation based on gravity and inertial effects. Moreover, humanoids have usually to ensure a firm contact with the ground, which provides reliable information on the surrounding environment. We show in this study, how important it is to take into account these information to improve IMU-based position/orientation reconstruction. We use an extended Kalman filter to rebuild the deformation, making the fusion between IMU and contact information, and without making any assumption on the dynamics of the flexibility. We show how, with this simple setting, we are able to compensate for perturbations and to stabilize the end-effector's position/orientation in the world reference frame.
\end{abstract}

\section{PROBLEM STATEMENT}

Many current humanoid robots are controlled as rigid systems, even if there are compliant and flexible parts in it. A good example of such a system is the robot HRP-2. Between the ankle and the sole of the robot, there lies a flexible bush (see Fig 1), designed to absorb foot impacts in order to protect force sensors and leg actuators [1]. However, this flexible part acts also as an angular spring and generates important deviation of the whole body, including the center of mass $(\mathrm{CoM})$, which is not modeled in the rigid system.

Therefore, this flexibility can threaten the balance of the robot, for example if the deformation deviates the CoM enough. Moreover, it also may jeopardize environment-related tasks. For example, in the case of drilling a wall, a robot has to apply forces on the wall. These forces will create a deformation of the compliant material and will deviate the robot's tool from its reference position and/or orientation.

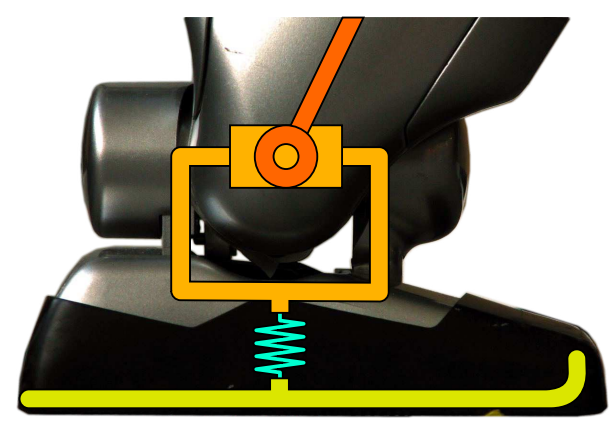

Fig. 1. The foot of HRP-2. Between the ankle joint and the sole of the robot, there is a rubber bush.

The problem of HRP-2 flexibility is currently tackled by a robot stabilizer. The stabilizer drives the deformation of the flexible

*This work was partially funded by the French Romeo-2 project

Mehdi Benallegue (mehdi@benallegue.com) and Florent Lamiraux (florentelaas.fr) are with:

${ }^{1}$ CNRS, LAAS, 7 avenue du colonel Roche, F-31400 Toulouse, France

2 Univ de Toulouse, LAAS, F-31400 Toulouse, France material to produce the desired forces and moments at feet, using a model of elasticity of the flexible material [2], [3]. At the same time, the upper-body orientation is maintained vertical, in order to minimize unwanted angular momentum and to enable to respect orientation-related upper-body tasks (manipulation, gaze, etc.). The orientation of the upper body is obtained using an inertial measurement unit at the chest of the robot. However, with this stabilizer, in the case of upper-body tasks, even if the torso is maintained upright, there is no guarantee that upper-body limbs are at their reference position, which is usually important when the robot is interacting with environment.

Moreover, the use of controllers based on force/torque sensors raises two main issues. First, these solutions are sensitive to sensors' calibration errors [4], which can even appear during operation of the robot (e.g. due to impacts, under constraints, etc). Second, these approaches can obviously not apply to robots which are not equipped with these expensive force sensors at contact points, such as the future Aldebaran's Romeo robot [5].

By contrast, inertial measurement sensors are cheap, relatively reliable and more robust. In addition, they provide important data on the real kinematics of the robot in the inertial frame. We show in this paper that, if we couple these sensors with contact point positions, we can afford a real-time fine estimation of the flexibility state, without any model of its compliance dynamics. We show also that these measurements can enable a stabilization of the robot's end-effector in the presence of external perturbations.

This study aims at proving the efficiency of inertial sensors for whole body pose estimation, and perturbations detection. In the next section we describe the theoretical guarantees and improvements provided by contact information to IMU measurements. The section III presents the demonstrative example of an implementation of such an observer with minimum prior knowledge. The section IV presents an experimental setting where we use our method for endeffector stabilization and compensation of perturbations. Finally the section $\mathrm{V}$ concludes the paper.

\section{THEORETICAL GROUNDING}

In the case of humanoid locomotion, the contact forces have usually to respect center of pressure and friction cone constraints in order to maintain balance [6]. That means that the contacts are firmly fixed to the environment. However, most methods for humanoid-robots attitude-estimation using inertial measurements do not consider contacts information, even for stabilizing the robots on their feet [7], [8]. Fixed contact positions provide coupling between the rotations and translations, transforming the inertial measurement unit (IMU) into a much more efficient sensor for reconstructing position/orientation. We show next a simple example of what this coupling provides to a simple pendulum.

\section{A. The inverted pendulum}

Let's consider a simple 3D inverted pendulum of $1 \mathrm{~m}$ length, connected to the ground with a 3 DoF ball joint, and with an 
inertial measurements unit (IMU), rigidly aligned at the top of the pendulum (see Figure 2). The configuration of the pendulum is the rotation matrix $R$. This orientation defines the position $\mathbf{p}$ of the IMU in the global frame:

$$
\mathbf{p}=R \mathbf{e}_{\mathbf{z}}
$$

where $\mathbf{e}_{\mathbf{z}}=\left[\begin{array}{lll}0 & 0 & 1\end{array}\right]^{t}$ is the unit vector along vertical $\mathbf{z}$ axis. If we consider that an unknown external source $\mathbf{u}$ provides the control of the pendulum's acceleration, we can write the following state dynamics:

$$
\dot{\mathbf{x}}=\left[\left([\omega]_{\times} R\right)^{t} \quad \dot{\omega}^{t} \quad 0\right]^{t}+\left[\begin{array}{lll}
0 & 0 & I
\end{array}\right]^{t} \mathbf{u}
$$

where $\mathbf{x}=\left[\begin{array}{lll}R^{t} & \omega^{t} & \dot{\omega}^{t}\end{array}\right]^{t}$ is the state vector formed by orientation, the angular velocity vector and the angular acceleration ${ }^{1}$, and $[\cdot]_{\times}$is the skew symmetric operator such that

$$
\left[\begin{array}{l}
x \\
y \\
z
\end{array}\right]_{\times}=\left[\begin{array}{ccc}
0 & -z & y \\
z & 0 & -x \\
-y & x & 0
\end{array}\right]
$$
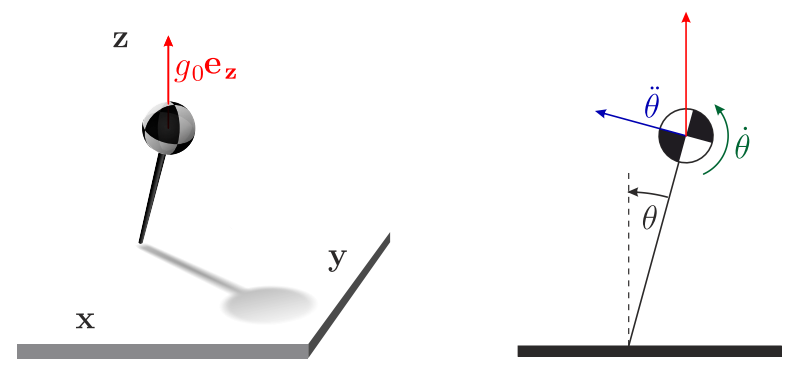

Fig. 2. On the left, 3D inverted pendulum. On the right, 2D inverted pendulum

The IMU at the top is composed of a gyrometer providing the angular rate at local reference frame $\mathbf{y}_{\mathbf{g}}$ and an accelerometer providing the gravity and the linear acceleration at the local frame $\mathbf{y}_{a}$. Let $\mathbf{y}=\left[\begin{array}{ll}\mathbf{y}_{g}^{t} & \mathbf{y}_{a}^{t}\end{array}\right]^{t}$ be the whole measurements vector:

$$
\mathbf{y}=\left[\begin{array}{c}
R^{t} \omega \\
R^{t}\left(\ddot{\mathbf{p}}+g_{0} \mathbf{e}_{\mathbf{z}}\right)
\end{array}\right]
$$

with $g_{0}$ the standard gravity constant. This measurement provides partial information on the configuration, but we show next that depending on whether we consider translations-orientations coupling or not, the observable parts of the vector differ significantly.

\section{B. Without translations-rotations coupling}

In the case we do not take into account the coupling, we have to consider that the linear acceleration $\ddot{\mathbf{p}}$ is a free input to the system for which we do not have any model. So, let's consider the case of an input acceleration $\ddot{\mathbf{p}}=-g \mathbf{e}_{\mathbf{z}}$, we have then $\mathbf{y}_{a}^{t}=0$, and the measurements provide only angular velocities, which are insufficient to reconstruct the orientation. Therefore, the configuration of the pendulum itself is not observable with these hypotheses.

Indeed, most today's approaches to reconstruct orientations with a gyrometer/accelerometer sensors without contact information, have to put an erroneous model on the linear acceleration, for example considered as Gaussian white noise for Kalman Filtering [9] or as high-frequency signal for complementary filtering [10].

\footnotetext{
${ }^{1}$ For simplicity, we keep the matrix representation of the orientation despite the heterogeneous nature of this state vector, in the next section we use rotation vector representation for the actual implementation.
}

These method lead to ignore translation accelerations, which may carry important and redundant information on the dynamics of the pendulum.

\section{With translations/rotations coupling}

If we use the rotations/translations coupling provided by Equation (1), we have then

$$
\mathbf{y}=\left[\begin{array}{c}
R^{t} \omega \\
{\left[R^{t} \dot{\omega}\right]_{\times} \mathbf{e}_{\mathbf{z}}+\left[R^{t} \omega\right]_{\times}^{2} \mathbf{e}_{\mathbf{z}}+g_{0} R^{t} \mathbf{e}_{\mathbf{z}}}
\end{array}\right]
$$

and let's consider the first derivative of the gyrometer measurements

$$
\dot{\mathbf{y}}_{g}=R^{t} \dot{\omega}
$$

with these three vectors, $\mathbf{y}_{g}, \mathbf{y}_{a}$ and $\dot{\mathbf{y}}_{g}$, we can reconstruct $R^{t} \mathbf{e}_{\mathbf{z}}$ and then the roll and pitch components of the configuration $R$. The yaw is unfortunately not observable with these sensors when there is only one contact with environment, since the system is invariant with respect to rotations around the $\mathbf{z}$ axis.

\section{The case of multiple contacts}

If the number of contacts between the sensor and the environment is 3 or more, the sensor is fully constrained and cannot move. So let's consider the 2 contacts situation. There remains only one degree of freedom, which is the rotation around the axis $\left(c_{1} c_{2}\right)$ passing by the two contact points. The system is equivalent to a $2 \mathrm{D}$ pendulum for which the configuration is defined only by one angle $\theta$, and the state becomes $x=\left[\begin{array}{lll}\theta & \dot{\theta} & \ddot{\theta}\end{array}\right]$ (see Figure 2). There are two possibilities, (i) the axis $\left(c_{1} c_{2}\right)$ is vertical, and in that case, the sensors can observe only angular velocities and accelerations around the axis, or (ii) the contact points are not vertically aligned and, without loss of generality, we can consider them at the same height and that the IMU is at 1 meter from the axis. If it is not the case, we only need to project the dynamics on the plane orthogonal to the axis $\left(c_{1} c_{2}\right)$ with minor adaptations to find the same developments.

The measurements vector is three-dimensional: angular velocity around the axis $\left(c_{1} c_{2}\right)$, and bi-dimensional accelerations orthogonal to it. All other measurements have constant values, the new measurement vector becomes:

$$
\mathbf{y}=\left[\begin{array}{c}
\dot{\theta} \\
\ddot{\theta}+g_{0} \sin (\theta) \\
\dot{\theta}^{2}+g_{0} \cos (\theta)
\end{array}\right]
$$

which can straightforwardly reconstruct all the state vector.

We see with the pendulum example that rotations/translation coupling, deduced from contact points information, can be taken into account to observe the sensor's attitude and position, without resorting to erroneous modeling of the state dynamics. In the next section, we show how we use this idea to reconstruct the state of the flexible part of the robot.

\section{FLEXIBILITY DEFORMATION OBSERVATION}

\section{A. Modeling the flexibility}

HRP-2 is a $30+6$ DoF robot controlled as being perfectly rigid. The configuration $\mathbf{q}$ is supposed to define perfectly for each limb $i$ the position/orientation in the world, represented by a homogeneous transformation matrix ${ }^{\mathcal{C}} M_{i}$ (C superscript is for "control").

However, there is a flexible part in HRP-2 which is a small compliant material between the sole and the ankle joint of the robot. It can be compressed, bent and twisted according to applied forces/moments. Therefore, when the robot is on its feet, the compliance modifies the configuration of the robot in rotations and 
translations, even when the contacts are balanced and immobile. We depict the flexibility deformation by a 6 DoF transformation represented by a homogeneous transformation matrix

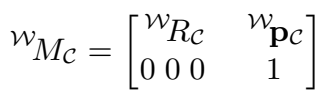

with $\mathcal{W}_{R_{\mathcal{C}}}$ and $\mathcal{W}_{\mathbf{p}_{\mathcal{C}}}$ are the rotation matrix and translation vector associated to the flexibility deformation.

Any limb $i$ at position/orientation ${ }^{{ }^{C}} M_{i}$ in the "control" reference

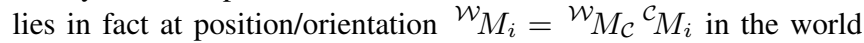
actual reference frame (see Figure 3). Therefore, ${ }^{\mathcal{W}_{M}} M_{\mathcal{C}}$ is not the matrix associated to a sole-ankle joint, but as a virtual joint between the world and the free-flier root joint of the robot. The choice of this representation enables to remain adapted to any number of supports and to guarantee continuity regardless of changes in contacts.

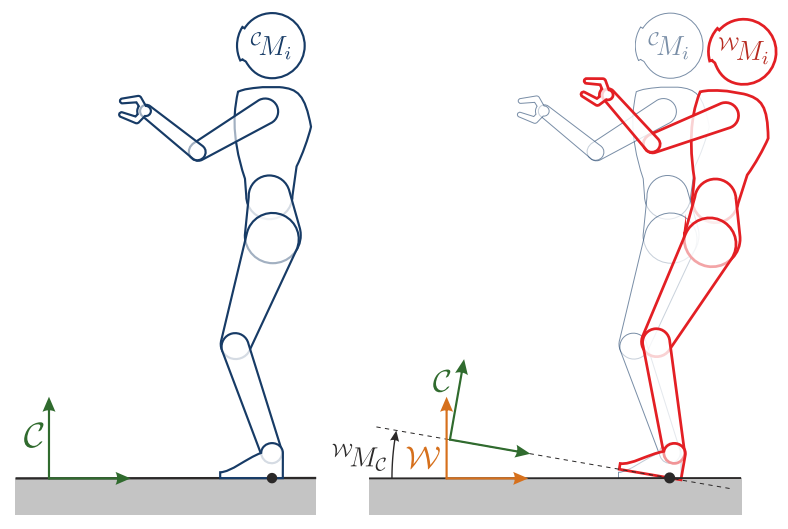

Fig. 3. On the left the rigid robot model in the "control" reference frame and the head position at $\mathcal{C}_{M}$. On the right, the flexibility $\mathcal{W}_{M_{\mathcal{C}}}$ transforms the configuration of the robot and the real position of the head $\mathcal{W}_{M}=\mathcal{W}_{M_{\mathcal{C}}} \mathcal{C}_{M}$ (in red) in the world reference frame. We see that $\mathcal{W}_{M_{\mathcal{C}}}$ is composed of a rotation and a translation.

To simplify notations, we omit the world frame $\mathcal{W}$ upper left superscript for next developments. Let's then define the 18 dimensional second order state vector $\mathbf{x}=$ $\left[\begin{array}{llllll}\mathbf{p}_{\mathcal{C}}^{t} & \Omega_{\mathcal{C}}^{t} & \dot{\mathbf{p}}_{\mathcal{C}}^{t} & \omega_{\mathcal{C}}^{t} & \ddot{\mathbf{p}}_{\mathcal{C}}^{t} & \dot{\omega}_{\mathcal{C}}^{t}\end{array}\right]^{t}$ where $\Omega_{\mathcal{C}}$ is the rotation vector representation of $R_{\mathcal{C}}$, such that $\exp \left(\left[\Omega_{\mathcal{C}}\right]_{\times}\right)=R_{\mathcal{C}}$, (i.e. $\left\|\Omega_{\mathcal{C}}\right\|$ is the angle or rotation and $\frac{\Omega_{\mathcal{C}}}{\left\|\Omega_{\mathcal{C}}\right\|}$ is the axis) and $\omega_{\mathcal{C}}$ is the vector of angular velocity.

Our study aims at showing that the measurements alone are able to provide accurate estimation of the flexibility state. Hence, we do not model the response of the flexibility to external forces. Instead we take the model of constant acceleration $\ddot{M}_{\mathcal{C}}$, which is a classical choice for pose and attitude estimation [11], [12], [13]. The discretetime model of the state dynamics is then:

$$
\mathbf{x}_{k+1}=f\left(\mathbf{x}_{k}\right)+\mathbf{v}_{k}
$$

where $f$ is a simple integrator with constant accelerations and $v_{k}$ is Gaussian white noise which is used to model the differences between the real dynamics of the state and the constant-acceleration model.

It is important to note that this dynamical system is chosen only for demonstration purposes, so it is deliberately erroneous and unstable. In fact, for a real use of the proposed observer, we suggest to replace this dynamical model by a model that is closer to the natural dynamics of the studied system, for example by a spring/damper or an inverted pendulum dynamical models. This would increase the precision of the estimation as modeling error is reduced.

\section{B. The sensors system}

This system is not stable and relies entirely on the measurements to correct it. We use the stock IMU sensor in HRP-2 which is located at the chest of the robot, and is composed of an accelerometer and a gyrometer. The measurement vector is then classically:

$$
\mathbf{y}=\left[\begin{array}{l}
\mathbf{y}_{g} \\
\mathbf{y}_{a}
\end{array}\right]=\left[\begin{array}{c}
R_{s}^{t} \omega_{s} \\
R_{s}^{t}\left(\ddot{\mathbf{p}}_{s}+g_{0} \mathbf{e}_{\mathbf{z}}\right)
\end{array}\right]
$$

where $\mathbf{y}_{g}$ and $\mathbf{y}_{a}$ are gyrometer and accelerometer measurements, and $R_{s}, \omega_{s}$ and $\mathbf{p}_{s}$ are respectively the rotation matrix, the angular velocity vector and the position of the IMU in the world reference frame.

We suppose that we know perfectly the robot's configuration $\mathbf{q}$ and its derivatives, we know then the position ${ }^{\mathcal{C}} R_{s}$, the angular velocity ${ }^{\mathcal{C}} \omega_{s}$, the position ${ }^{\mathcal{C}} \mathbf{p}_{s}$ of the IMU, and their derivatives, in the control reference frame. On the other hand, we have the relationships due to flexibility: $R_{s}=R_{\mathcal{C}}{ }^{\mathcal{C}} R_{s}$ and $\mathbf{p}_{s}=R_{\mathcal{C}}{ }^{\mathcal{C}} \mathbf{p}_{s}+$ $\mathbf{p}_{\mathcal{C}}$. All that leads to:

$$
\begin{aligned}
& \mathbf{y}_{\mathbf{g}}={ }^{\mathcal{C}} R_{s}^{t}{ }^{\mathcal{C}} \omega_{s}+{ }^{\mathcal{C}} R_{s}^{t} R_{\mathcal{C}}^{t} \omega_{\mathcal{C}} \\
& \mathbf{y}_{\mathbf{a}}={ }^{\mathcal{C}} R_{s}^{t} R_{\mathcal{C}}^{t}\left(\left(\left[\dot{\omega}_{\mathcal{C}}\right]_{\times}+\left[\omega_{\mathcal{C}}\right]_{\times}^{2}\right) R_{\mathcal{C}}{ }^{\mathcal{C}} \mathbf{p}_{s}+2\left[\omega_{\mathcal{C}}\right]_{\times} R_{\mathcal{C}}{ }^{{ }^{C}} \dot{\mathbf{p}}_{s}\right) \\
& +{ }^{\mathcal{C}} R_{s}^{t}\left({ }^{\mathcal{C}} \ddot{\mathbf{p}}_{s}+R_{\mathcal{C}}^{t} \ddot{\mathbf{p}}_{\mathcal{C}}\right)+g_{0}{ }^{\mathcal{C}} R_{s}^{t} R_{\mathcal{C}}^{t} \mathbf{e}_{\mathbf{z}}
\end{aligned}
$$

But because the measurements are noisy and our dynamics is discretized, we model the sensors dynamics as following

$$
\mathbf{y}_{k}=g\left(\mathbf{x}_{k}, \mathbf{q}_{k}, \dot{\mathbf{q}}_{k}, \ddot{\mathbf{q}}_{k}\right)+\mathbf{w}_{\mathbf{s} k}
$$

Where $g$ is the measurement function summarizing equations (10) and (11) and $\mathbf{w}_{\mathbf{s} k}$ is a Gaussian white noise.

\section{The contact points}

We have seen that the flexibility provides six supplementary degrees of freedom to the robot. However, the size of the flexible bush and the magnitude of the linear forces exerted on it make linear translations of the compliance almost negligible. In other words, we can consider that globally the contact point positions in the world and in the control reference frame are almost identical: $M_{\mathcal{C}}{ }^{C} \mathbf{p}_{c_{i}} \simeq{ }^{\mathcal{C}} \mathbf{p}_{c_{i}}$, where ${ }^{\mathcal{C}} \mathbf{p}_{c_{i}}$ is the position of the $i$-th contact in the control reference frame, which is considered as perfectly known for all the contacts. We put then an approximation instead of an equality constraint. This enables to leave some freedom to violate the constraint and to detect for example sideways perturbations during double support. Thus, instead of constraining the contact points to be fixed to environment, we add fake measurements to our dynamical system. These measurement claim that the contacts are fixed in environment $M_{\mathcal{C}}{ }^{C} \mathbf{p}_{c_{i k}}-{ }^{\mathcal{C}} \mathbf{p}_{c_{i k}}=0$, but we model these measurements as being noisy to allow some freedom. So for each contact $c_{i}$, the sensors model is:

$$
\mathbf{r}_{\mathbf{i} k}=M_{\mathcal{C} k}{ }^{\mathcal{C}} \mathbf{p}_{c_{i k}}-{ }^{\mathcal{C}} \mathbf{p}_{c_{i k}}+\mathbf{w}_{\mathbf{i} k}
$$

where $\mathbf{w}_{\mathbf{i} k}$ is a Gaussian white noise.

We can gather then the measurements of a $n$ contacts configuration in a $6+(3 \times n)$ dimensional vector:

$$
\mathbf{z}_{k}=h\left(\mathbf{x}_{k}, \mathbf{q}_{k}, \dot{\mathbf{q}}_{k}, \ddot{\mathbf{q}}_{k},{ }^{\mathcal{C}} \mathbf{p}_{c_{1 k}}, \ldots,{ }^{C} \mathbf{p}_{c_{n k}}\right)+\mathbf{w}_{k}
$$

where $h$ stacks all the measurements described earlier (IMU + fake measurements) and $\mathbf{w}_{k}$ stacks all the measurement noises.

It is worth mentioning that, for the continuous-time system, the measurements described here provide the same observability 
properties as the inverted pendulum example of the previous section. In the case of one contact, the orientation is observable except in yaw, which leads to a partial observability of the position $\mathbf{p}_{\mathcal{C}}$ (constrained to a circle around the vertical line passing by the contact point). In the case of multiple contacts, everything is observable.

\section{Extended Kalman Filtering}

We use for the estimation of the state vector a classical Extended Kalman Filter (EKF). This observer works in two steps for each time sample: the prediction and the update.

1) Prediction: Let's suppose that at instant $k+1$, we have already an estimation of $\mathbf{x}_{k}$ which we denote $\hat{\mathbf{x}}_{k}$. We model the error $\mathbf{e}_{k}=\mathbf{x}_{k}-\hat{\mathbf{x}}_{k}$ as a random variable following a centered Gaussian distribution for which we suppose that we know the covariance matrix $P_{k}$. The prediction consists in simulating the modeled dynamics of the system if it were neither noisy nor perturbed. We define:

$$
\overline{\mathbf{x}}_{k+1}=f\left(\hat{\mathbf{x}}_{k}\right)
$$

where $\overline{\mathbf{x}}_{k+1}$ is the predicted state. This prediction commits also an error $\overline{\mathbf{e}}_{k+1}=\mathbf{x}_{k+1}-\overline{\mathbf{x}}_{k+1}$ which is due to the transport of $\mathbf{e}_{k}$ by $f$ in addition to the process noise $\mathbf{v}_{k+1}$. We model also the error $\overline{\mathbf{e}}_{k+1}$ as a Gaussian random variable and we linearize $f$ to approximate its covariance matrix:

$$
\bar{P}_{k+1}=F_{k+1} P_{k} F_{k+1}^{t}+Q_{k+1}
$$

where $Q_{k+1}$ is the covariance matrix of $\mathbf{v}_{k+1}$, and

$$
F_{k+1}=\left.\frac{\partial f}{\partial \mathbf{x}}\right|_{\mathbf{x}=\hat{\mathbf{x}}_{k}}
$$

This prediction enables to estimate the measurements obtained if the state was equal to the prediction:

$$
\overline{\mathbf{z}}_{k+1}=h\left(\overline{\mathbf{x}}_{k+1}, \mathbf{q}_{k+1}, \dot{\mathbf{q}}_{k+1}, \ddot{\mathbf{q}}_{k+1},{ }^{\mathcal{C}} \mathbf{p}_{c_{1 k+1}}, \ldots,{ }^{\mathcal{C}} \mathbf{p}_{c_{n k+1}}\right)
$$

where $\overline{\mathbf{z}}_{k+1}$ is the predicted measurement vector.

2) Update: The update consists in using the actual measurements to correct, to some extent, the prediction of the previous step. The actual measurements are in our case the vector $\mathbf{z}_{k+1}=$ $\left[\begin{array}{lll}\mathbf{y}_{\mathbf{g}_{k+1}}^{\mathbf{t}} & \mathbf{y}_{\mathbf{a}}^{\mathbf{t}} & 0_{1 \times 3 n}\end{array}\right]^{t}$. We define the measurements innovation:

$$
e_{\mathbf{z} k+1}=\mathbf{z}_{k+1}-\overline{\mathbf{z}}_{k+1}
$$

Again, this error is modeled as a Gaussian random variable, and the covariance matrix is approximated as follows:

$$
P_{\mathbf{z} k+1}=H_{k+1} \bar{P}_{k+1} H_{k+1}^{t}+R_{k+1}
$$

where $R_{k+1}$ is the covariance matrix of $\mathbf{w}_{k+1}$, and

$$
H_{k+1}=\frac{\partial h}{\partial \mathbf{x}} \mid \begin{aligned}
& \mathbf{x}=\overline{\mathbf{x}}_{k+1}, \mathbf{q}=\mathbf{q}_{k+1}, \dot{\mathbf{q}}=\dot{\mathbf{q}}_{k+1}, \ddot{\mathbf{q}}=\ddot{\mathbf{q}}_{k+1}, \\
& \mathcal{C}_{\mathbf{p}_{c_{1}}}=\mathcal{C}_{\mathbf{p}_{c_{1}+1}}, \ldots, \mathbf{p}_{c_{n}}=\mathcal{C}_{\mathbf{p}_{n}}, \\
& c_{n+1}
\end{aligned}
$$

This enables to compute the near-optimal gain $K_{k+1}$ minimizing the quadratic error expectation for the estimation error $\mathbf{e}_{k+1}=$ $\mathbf{x}_{k+1}-\hat{\mathbf{x}}_{k+1}$, where

$$
\hat{x}_{k+1}=\bar{x}_{k+1}+K_{k+1} e_{\mathbf{z} k+1}
$$

The near-optimal gain $K_{k+1}$ is obtained as follows:

$$
K_{k+1}=\bar{P}_{k+1} H_{k+1}^{t} P_{\mathbf{z}_{k+1}}^{-1}
$$

And the corresponding covariance matrix of $\mathbf{e}_{k+1}$ is then

$$
P_{k+1}=\left(I-K_{k+1} H_{k+1}\right) \bar{P}_{k+1}
$$

The size of the measurements vector $\mathbf{z}_{k}$ may change if a contact on environment is removed or added, but this does not change the state value nor the extended Kalman filter developments.

We have now an estimator of the flexibility deformation in its $6 \mathrm{DoF}$ and its derivatives. This estimation can be directly used for example in order to correct the position of the end effector as we show in the next section.

\section{HAND POSITION COMPENSATION}

\section{A. The hand position in the world frame}

When a humanoid robot interacts with the environment, the endeffector has usually to follow trajectories in the control reference frame. We have seen that because of flexible parts of the robot, these trajectories may differ in the world reference frame. Moreover, if the robot has to exert forces on the environment in some tasks, such as drilling a wall, the reaction force will create a deformation of the flexible parts and will move the end effector from its reference position.

We propose here to show a direct use of the flexibility observer. We put HRP-2 on its feet, we ask the robot to keep the right hand at a given reference position/orientation, summarized in a homogeneous matrix $M_{r}$, and we push the robot to excite flexibility. However, the hand controller takes only references expressed in $\mathcal{C}$. So, the classical solutions consists in working in the control reference frame $\mathcal{C}$, giving a reference ${ }^{\mathcal{C}} M_{r}=M_{r}$. Obviously the hand will swing with all the robot, and will not keep its reference position, when the flexibility is deformed. Instead, we propose to ask for another reference of hand position and orientation ${ }^{\mathcal{C}} M_{r}=M_{\mathcal{C}}^{-1} M_{r}$, with the flexibility deformation $M_{\mathcal{C}}$ estimated using the extended Kalman filter described in the prevous section. If the flexibility is efficiently reconstructed, the hand will stay at the same position in the world reference frame (see Figure 4).

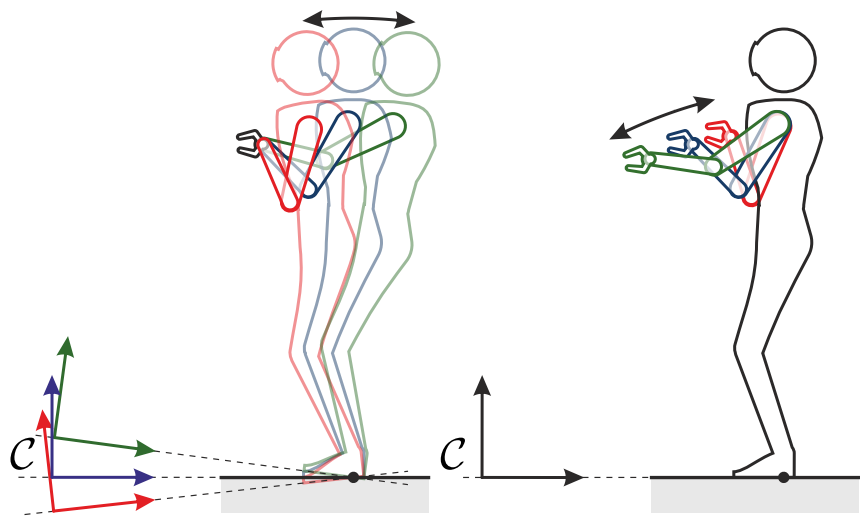

Fig. 4. On the left, the hand compensation in the world reference frame On the right what happens at the same time in the control reference frame.

\section{B. Experimental setting}

To achieve this setting, we use the Stack of Tasks framework [14], which is a task-based hierarchical inverse kinematics solver. The tasks were set, in a decreasing priority order, to (i) keep both feet on the ground and the center of mass above the middle of them, in the control reference frame (ii), keep the height ${ }^{{ }} \mathbf{p}_{t}$ and the orientation ${ }^{{ }^{C}} R_{t}$ of the trunk (waist, chest and head), (iii) keep the right hand at ${ }^{\mathcal{C}} M_{r}={ }^{\mathcal{W}_{M}} M_{\mathcal{C}}^{-1} M_{r}$ and (iv) reproduce with the left hand the motion of the right hand. The last task is just to play the role of counterweight and avoid unstable dynamical effects of hand compensation. 
The stack of tasks (SoT) enables also to introduce a feed-forward term which is the desired velocity of the task in the control reference frame. We introduce then also the following desired hand linear and angular velocities

$$
\begin{aligned}
& { }_{\dot{\mathbf{p}}_{r}}=\left[R_{C}^{t} \omega_{C}\right]_{\times} R_{C}^{t}\left(\mathbf{p}_{C}-\mathbf{p}_{r}\right)-R_{C}^{t} \dot{\mathbf{p}}_{C} \\
& { }^{\mathcal{c}} \omega_{r}=-R_{C}^{t} \omega_{C}
\end{aligned}
$$

where $p_{r}$ is the reference position of the hand in the world reference frame.

To show the performances of the observer, three experiments were conducted:

1) the reference orientation of the trunk ${ }^{\mathcal{C}} R_{t}$ is constant at upright position, and the robot is pushed to excite flexibility. The expected result is that the hand stays at the reference position while the whole robot is displaced.

2) the reference orientation of the trunk ${ }^{{ }^{C}} R_{t}$ oscillates in time. The IMU is then excited and provides oscillating measurements. The flexibility is excited as well, due to torques that move the upper-body, but the excitation is of much smaller magnitude than when the robot is pushed.

3) the oscillation of the trunk and the external perturbations are combined. The estimator has to distinguish between the signals due to the oscillation and those due to flexibility deformation.

\section{Results}

During the first experiment, the external perturbations made the robot oscillate of up to $0.175 \mathrm{rad}$ (about $10^{\circ}$ ). The hand position, being at $1.1 \mathrm{~m}$ distance to the contact point, if it was not compensated, would move by about $20 \mathrm{~cm}$. Instead, the hand moves by less than $2.0 \mathrm{~cm}$. During the second experiment, the flexibility was slightly excited and observed by the Kalman filter. However the oscillation of the upper-body created vibration, detected particularly by the gyrometer. which lead to small estimation error, the hand moved by about $1.5 \mathrm{~cm}$. During the third experiment, the combination of oscillation and the perturbation did not degrade the performances and the hand moved still by less than $2.0 \mathrm{~cm}$.

The results are summarized in Fig. 5, and the relevant signals and observations in Fig. 6. The video attachment presents also the performances under several angles, including the ankle of the robot, and shows responses to lateral perturbations. We remind that only the right hand is stabilized and the left hand is just a counterweight.

\section{DISCUSSION AND CONCLUSION}

We have seen through this paper that the contact with environment enables to take profit from the position-orientation coupling, in order to better distinguish between body accelerations and gravitational component in accelerometer measurements. The separation of these two signals have two benefits: it increases the precision in verticality estimation (pitch and roll), but also it provides information on the acceleration of the attitude and position.

The combination of the inertial measurements and legs kinematics is already a subject or active research, but almost exclusively on multi-legged robots for odometry and localization purposes [15], [16], [17], [18], [19], [20]. These methods provide reliable information on the position in the world. However, none of them took profit of the accelerations-gravity decoupling, even when attitude reconstruction was an important component of the reconstructed state vector [19], [20].

To our best knowledge, only one method takes this advantage, which is presented by Bloesch et al. [21] for a multi-legged
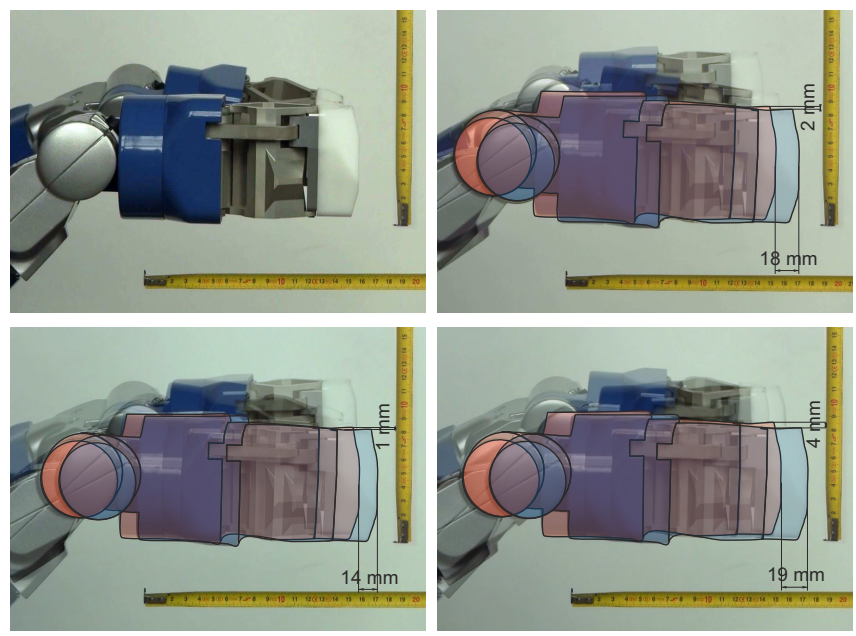

Fig. 5. In the top left, a screen capture of the robot's hand during the compensation experiment. In the top right a superimposition of the two pictures of the extreme points of hand stabilization during the excitation of the flexibility. In the bottom left, a superimposition of extreme positions during oscillation. And in the bottom right, a superimposition of extreme positions during combined oscillation and excitation of the flexibility. For each superimposition, the position displacement for the hand is shown.

robot. They used the contact information provided by proprioceptive sensors and kinematics model and merged them with IMU signals in an multiplicative/additive extended Kalman filter. They could then constrain the dynamics of their state to respect contact kinematics, and use translations-rotation coupling to improve their estimation. However, their model considers the IMU signals as inputs to the system and not measurements. So their model of the dynamics is an integration of the measurements. This prevents having another model of the state dynamics (e.g. inverted pendulum, spring damper, etc) because this would remove IMU information from models. They also consider slipping contacts as measurements, which may be adapted to walking on uncertain environments, but which leads to the non-observability of the position as aknowledged by the authors. That means that the position may drift for long observation periods. This would lead for example the hand compensation experiment to deviate eventually from the reference positions. This issue could possibly be partially solved by reducing the covariance of the noise model of the measurements, but Kalman filtering is likely to have numerical issues with too certain or perfect measurements [22]. In fact, for humanoid robots in known environments, we can consider the contacts as firmly linked to the ground and this should be exploited to constrain the dynamics of the reconstructed attitude, as presented in our study. Finally, Bloesch et al. model a rigid contact with the ground while our model enables small deviation in translation from the contact point. This deviation may happen in the case of a flexibility in translation, but this is different from slipping contact because in average the contact position is assumed constant, as it is guaranteed by our observer.

Some issues may raise and be considered as limitations of our approach. First, if there is more than one flexible part in the robot, one single IMU does not guarantee the observability of the flexibility. In that case, a solution may be to use several IMUs [23]. Second, in the case the joint positions are not perfectly known, the estimations error would be proportional to errors in contact positions and to errors in the position/orientation of the IMU and their derivatives in the control frame. 

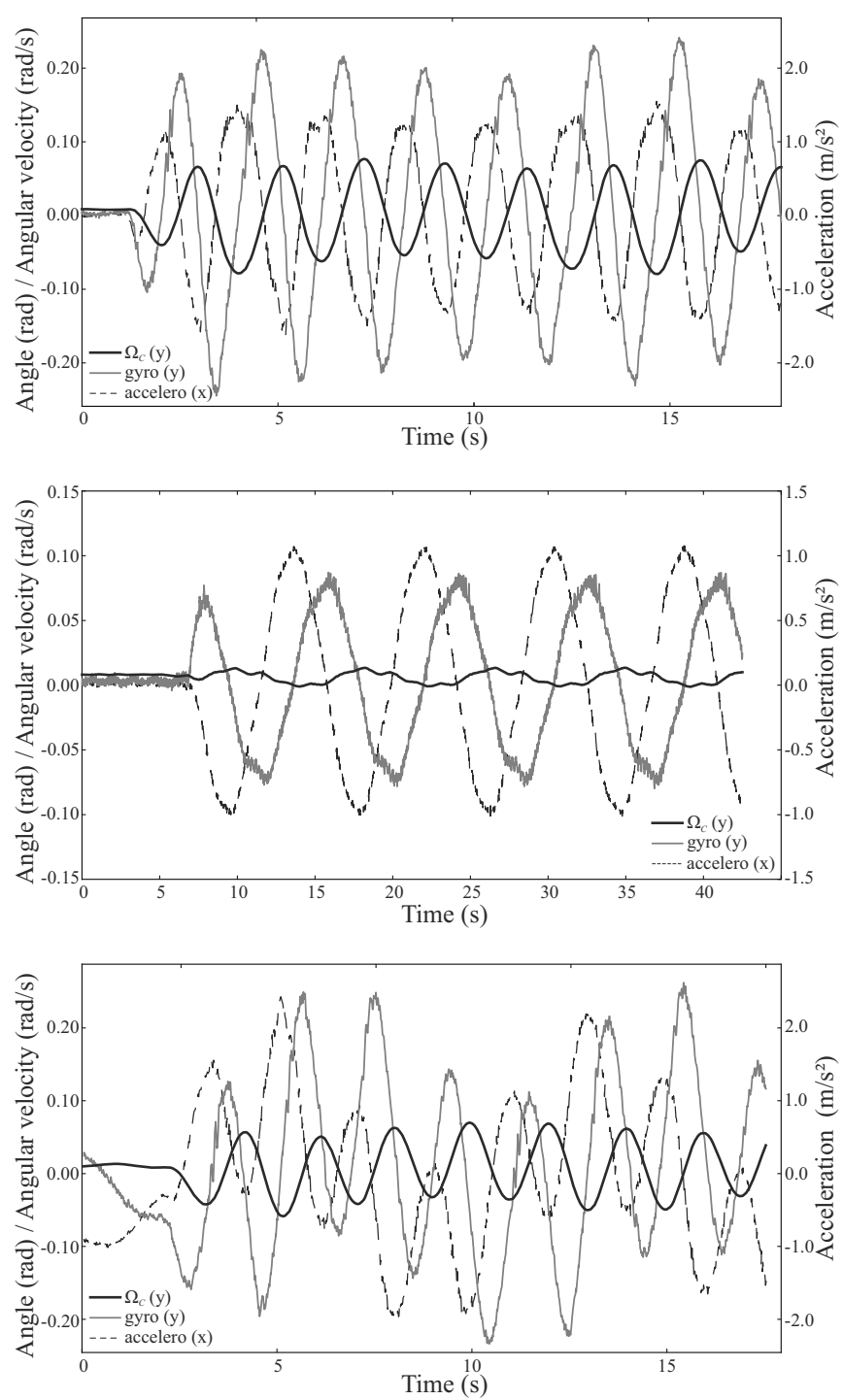

Fig. 6. Signals of accelerometer along $\mathrm{x}$, the gyrometer around $\mathrm{y}$, and flexibility orientation $\Omega_{\mathcal{C}}$ around $y$, in the case of the three experiments. In top, the first experiment (the external perturbations on the upright robot). In middle, the second experiment (the robot's trunk is oscillating without external perturbations). In bottom, the third experiment (trunk oscillation and external perturbations are combined).

Finally, the main purpose of a flexibility-deformation stateobserver should go beyond simply stabilizing end-effectors. Indeed, estimating efficiently the flexibility deformation means that (i) we know the actual kinematics of the center of mass in the world reference frame and (ii) we have an estimation of the ground reaction forces if we have a model of the force-response to deformations of the flexible part of the robot. Both estimations are precious information on the dynamic balance of the humanoid robot. Therefore, the main objective of this study is to open the way to balancing and stabilizing techniques that would not only run on robots that are not equipped by force sensors, but also provide redundant sensing to stabilization methods relying on force/torque sensors.

\section{REFERENCES}

[1] N. Kanehira, T. Kawasaki, S. Ohta, T. Ismumi, T. Kawada, F. Kanehiro, S. Kajita, and K. Kaneko. Design and experiments of advanced leg module (hrp-21) for humanoid robot (hrp-2) development. In Intelligent Robots and Systems, volume 3, pages 2455-2460, 2002.

[2] S. Kajita, K. Yokoi, M. Saigo, and K. Tanie. Balancing a humanoid robot using backdrive concerned torque control and direct angular momentum feedback. In ICRA, 2001.

[3] S. Kajita, T. Nagasaki, K. Kaneko, K. Yokoi, and K. Tanie. A running controller of humanoid biped hrp-21r. In Intl. Conf. Robotics and Automation, (ICRA)., pages 616-622. IEEE, 2005.

[4] R. M Voyles, J. D. Morrow, and P. K Khosla. The shape from motion approach to rapid and precise force/torque sensor calibration. Journal of dynamic systems, measurement, and control, 119(2):229-235, 1997.

[5] Aldebaran Robotics. Romeo. http://www.projetromeo.com, 2011.

[6] P.-B. Wieber. On the stability of walking systems. In Proceedings of the International Workshop on Humanoid and Human Friendly Robotics, Tsukuba, Japan, 2002.

[7] N. Perrin, N. Tsagarakis, and D. G. Caldwell. Compliant attitude control and stepping strategy for balance recovery with the humanoid coman. In Intelligent Robots and Systems (IROS), 2013, pages 41454151. IEEE, 2013.

[8] S. Kajita, M. Morisawa, K. Miura, S. Nakaoka, K. Harada, K. Kaneko, F. Kanehiro, and K. Yokoi. Biped walking stabilization based on linear inverted pendulum tracking. In Intelligent Robots and Systems (IROS), pages 4489-4496, Oct 2010.

[9] J. L. Crassidis, F. L. Markley, and Y. Cheng. Survey of nonlinear attitude estimation methods. Journal of Guidance, Control, and Dynamics, 30(1):12-28, 2007.

[10] V. Kubelka and M. Reinstein. Complementary filtering approach to orientation estimation using inertial sensors only. In Robotics and Automation (ICRA), pages 599-605, May 2012.

[11] B. Barshan and H. F Durrant-Whyte. Inertial navigation systems for mobile robots. Robotics and Automation, IEEE Transactions on, 11(3):328-342, 1995.

[12] P.-C. Lin, H. Komsuoglu, and D. E Koditschek. Sensor data fusion for body state estimation in a hexapod robot with dynamical gaits. Robotics, IEEE Transactions on, 22(5):932-943, 2006.

[13] O. Gür. and U. Saranli. Model-based proprioceptive state estimation for spring-mass running. In Proceedings of Robotics: Science and Systems, Los Angeles, CA, USA, June 2011.

[14] N. Mansard, O. Stasse, P. Evrard, and A Kheddar. A versatile generalized inverted kinematics implementation for collaborative working humanoid robots: The stack of tasks. In International Conference on Advanced Robotics, pages 1-6, June 2009.

[15] B. Gassmann, F. Zacharias, J.M. Zollner, and R. Dillmann. Localization of walking robots. In IEEE International Conference on Robotics and Automation, pages 1471-1476, April 2005.

[16] S. Chitta, P. Vernaza, R. Geykhman, and D.D. Lee. Proprioceptive localilzatilon for a quadrupedal robot on known terrain. In IEEE International Conference onRobotics and Automation, pages 45824587, April 2007.

[17] J.A. Cobano, J. Estremera, and P. Gonzalez de Santos. Location of legged robots in outdoor environments. Robotics and Autonomous Systems, 56(9):751 - 761, 2008

[18] P.-C. Lin, H. Komsuoglu, and D.E. Koditschek. Sensor data fusion for body state estimation in a hexapod robot with dynamical gaits. Robotics, IEEE Transactions on, 22(5):932-943, Oct 2006.

[19] A Chilian, H. Hirschmuller, and M. Gorner. Multisensor data fusion for robust pose estimation of a six-legged walking robot. In IEEE/RSJ International Conference on Intelligent Robots and Systems (IROS), pages 2497-2504, Sept 2011

[20] M. Reinstein and M. Hoffmann. Dead reckoning in a dynamic quadruped robot: Inertial navigation system aided by a legged odometer. In Robotics and Automation (ICRA), 2011 IEEE International Conference on, pages 617-624, May 2011.

[21] Michael Bloesch, Marco Hutter, Mark Hoepflinger, Stefan Leutenegger, Christian Gehring, C. David Remy, and Roland Siegwart. State estimation for legged robots - consistent fusion of leg kinematics and IMU. In Proceedings of Robotics: Science and Systems, Sydney, Australia, July 2012.

[22] Dan Simon and Tien Li Chia. Kalman filtering with state equality constraints. Aerospace and Electronic Systems, IEEE Transactions on, 38(1):128-136, 2002.

[23] Silvio Traversaro, Daniele Pucci, and Francesco Nori. In situ calibration of six-axes force torque sensors using accelerometer measurements. arXiv preprint arXiv:1410.0885, 2014 